\title{
Hydrodynamics and Theoretical Light Curves of SNe II
}

\author{
By V. S. IMSHEN NIK AND S. I. BLINNIKOV
}

ITEP, 117259 Moscow, Russia

We discuss a new scenario for the production of SN II explosion and present the results of numerical modelling studies of SNe II light curves which are being done in our group.

\section{Exploding Neutron Star}

The outburst of SN 1987A has given a powerful impetus for theoretical work on the physical mechanism of supernova explosions. The one-dimensional theory of the SN mechanism has met certain difficulties in explaining the SN II explosion (see, e.g. Imshennik 1992a). Multidimensional effects might be required to resurrect the delayed explosion mechanism (Bethe \& Wilson 1985), owing to neutrino heating (see contributions by Burrows 1993 and Janka 1993). Hillebrandt et al. (1990) have remarked that we may have to invent complicated scenarios in order to account for the explosions of massive stars, $M=20 M_{\odot}$. We discuss here a bizarre scenario proposed by Imshennik (1992b), where the interested reader can find further details. Here we give only a brief sketch of the main idea and report on the present status of the project.

In the suggested scenario (Imshennik 1992b), the decisive role is played by the rotation of a presupernova core. The idea to connect an SN explosion with the fission instability in a rapidly rotating collapsing star was first put forward by von Weizsäcker (1947). Shklovsky (1970) had also pointed out the possible importance of the rotational instability for type II SNe. Those ideas were expressed in quite general form. The scenario suggested by Imshennik (1992b) specifies the concrete form of the operation of the instability. We note that the magnetic field is not decisive in this scenario, unlike the mechanism of Bisnovatyi-Kogan (1970).

The collapse of the rotating core leads to the formation of a flattened, rapidly rotating protoneutron star. The fission of this configuration results in the formation of a close neutron star binary. Due to the gravitational radiation the binary components approach each other. After the low mass component fills its Roche lobe it is stripped by the high mass one. When its mass drops below the minimum value for a stable neutron star, $\sim 0.1 M_{\odot}$, it explodes with the energy release $\sim 10^{51}$ ergs.

To illustrate this scenario we use the numbers from the numerical model of rotating collapsing core computed by Imshennik \& Nadyozhin (1992), which is identical to the model used by Imshennik \& Nadyozhin (1977). The neutrino transport is treated there as in the work by Nadyozhin (1977) and the effects of rotation are averaged over the polar angle. The initial configuration is approximated by a uniformly rotating polytrope $n=3$ with mass $M_{\mathrm{t}}=2 M_{\odot}$, radius $R_{0}=4.38 \times 10^{8} \mathrm{~cm}$, and angular velocity $\omega_{0}=1.53 \mathrm{~s}^{-1}$. This choice of parameters gives the initial angular momentum $J_{0}=8.81 \times 10^{49} \mathrm{erg} \cdot \mathrm{s}$. If one assumes that this core collapses into one protoneutron star, then one gets the ratio of rotational to gravitational energy, $\eta=E^{\text {rot }} /\left|E^{\mathrm{g}}\right|=0.42$. It is well known that this value is well above the threshold of the dynamical instability $\eta \simeq 0.27$ (Tassoul 1979), so the outcome of the collapse is most probably the formation of a binary neutron star system. Assuming the conservation of mass and of angular momentum one finds for the 
semimajor axis of the system:

$$
a=J_{0}^{2} /\left\{G M_{\mathrm{t}}^{3}[\delta(1-\delta)]^{2}\right\}
$$

where $\delta=M_{1} / M_{\mathrm{t}}$, if one denotes the mass of components by $M_{1}$ and $M_{2}$. For the assumed parameters we find from (1.1):

$$
a=18.5[\delta(1-\delta)]^{-2} \mathrm{~km} .
$$

According to (1.2) the orbit radius $a$ falls in the range $300-500 \mathrm{~km}$ for the values of $\delta$ between $1 / 4$ and $3 / 4$. The bulk of neutrinos observed from SN $1987 \mathrm{~A}$ is provided by the more massive neutron star just at the moment of fission. The subsequent fate of the binary is determined by its gravitational radiation (cf. the analysis by Blinnikov et al. 1984). The timescale for decrease of the semimajor axis $a(\mathrm{~km})$ is

$$
t_{\text {grav }} \simeq 1.71 \times 10^{-7}\left(\frac{M_{\odot}}{M_{\mathrm{t}}}\right)^{3} \frac{a^{4}}{\delta(1-\delta)} \text { sec. }
$$

Merging of the components may produce the explosion as in some models for $\gamma$-ray bursts (e.g., Meszaros \& Rees 1992; Narayan, Paczyński \& Piran 1992; Woosley 1993a). Here we consider another possibility: the low mass component may be stripped by the high mass one. We follow the scenario discussed by Blinnikov et al. (1984) for old neutron star binaries in connection with their possible explosion and production of $\gamma$ ray bursts. When $a$ drops by a factor of 10 from its initial value in eq. (1.2) (this factor is virtually independent of the mass ratio $\delta$ ), the less massive component fills its Roche lobe. This circumstance may lead to an intensive mass transfer from the less massive component to the more massive one (Blinnikov et al. 1984). A neutron star with the mass $M<M_{\text {cr }} \simeq 0.1 M_{\odot}$ is dynamically unstable. So, when the less massive star loses enough mass, it must explode. The numerical modelling by Blinnikov et al. (1990) has demonstrated that the explosion leads to a remarkably constant energy release, $E_{\text {kin }} \simeq 8.8 \times 10^{50} \mathrm{ergs}(\sim 4.8 \mathrm{MeV} /$ nucleon $)$.

The gravitational timescale (1.3) is short enough to meet the restrictions posed by the neutrino observations of SN 1987A. Imshennik (1992b) shows that the time between the neutrino burst and the explosion may be less than $\sim 1$ hour if the parameter $\delta$ lies within reasonable limits: $0.3<\delta<0.7$. We note that the more massive companion may well collapse into a black hole, so after the less massive component explodes (the event takes place far away from the primary), there is no neutron star left.

Of course, there are many points in the above scenario which deserve confirmation at least by a simplified numerical modelling, and we have started a project intended to do so. The most difficult point is the fragmentation, or fission, of the rotating collapsing core. To simulate this phenomenon, one must perform 3-dimensional computations for gravitating fluid, taking into account the emission of neutrinos and gravitational waves. This is a grandiose task which may be left for future. At present we are testing a 3-dimensional hydrocode which is capable to treat a simplified problem of fission in Newtonian gravity (Aksenov \& Imshennik 1993).

The code realizes the Eulerian PPM scheme (Colella \& Woodward 1984). Unlike Imshennik \& Nadyozhin (1992), who have used the ideal gas approximation, Aksenov \& Imshennik (1994) rely on a more realistic equation of state which includes the non-ideal effects in neutron matter (Friedmann \& Pandharipande 1981). The real gas approximation implies the necessity to use appropriate solution algorithms for the Riemann problem (Colella \& Glas 1985) and to care about the initial model. The hydrocode provides exact conservation of mass and momentum. The energy is conserved exactly only in the absence of gravity. The Poisson equation is solved with the help of expansion in 
Legendre polynomials. This procedure is sufficiently efficient: one needs $\propto l_{\max }$ floating point operations per mesh point, where $l_{\max }$ is the highest order of Legendre polynomials used. The same efficiency is reached also in the general 3-dimensional case when one uses the associated Legendre polynomials (Aksenov 1993). The boundary condition for the gravitational potential, $\Phi \rightarrow-G M / r$ for $r \rightarrow \infty$, is automatically satisfied by this technique.

At present we have detailed calculations only with the 2-dimensional version of the code. The test problem solved is to calculate the hydrodynamical evolution of the rapidly rotating 1-dimensional body (obtained by Imshennik \& Nadyozhin 1992) in a more realistic 2-dimensional configuration (this is of course not fully realistic, since 3-dimensional effects can develop already at previous stages of collapse). The numerical solution is found on a $120 \times 64$ for $l_{\max }=28$ grid. The mesh is not uniform over radius $r$ : it is denser for $r<r_{\max } / 4$ (this region contains $2 / 3$ of all mesh zones). The difference scheme conserves mass and momentum exactly. The angular momentum is conserved with high accuracy, $\sim 10^{-4}$, and the total energy with accuracy better than $5 \%$.

We present here some results of Aksenov \& Imshennik (1994). As usual, we introduce the gravitational energy, $E^{\mathrm{gr}}=\frac{1}{2} \int \rho \Phi d \mathbf{r}$, and the kinetic energy, $E^{\mathrm{k}}=\frac{1}{2} \int \rho \mathbf{v}^{2} d \mathbf{r}$. Let us separate $E^{\mathrm{k}}$ into the rotational, $E^{\mathrm{rot}}$, and poloidal parts,

$$
E^{\mathrm{k} 0}=\frac{1}{2} \int \rho\left(v_{r}^{2}+v_{\theta}^{2}\right) d \mathbf{r}=E^{\mathrm{k}}-\frac{1}{2} \int \rho v_{\varphi}^{2} d \mathbf{r}=E^{\mathrm{k}}-E^{\mathrm{rot}}
$$

It is clear that in the case of pure steady rotation $E^{\mathrm{k} 0}=0$.

We present in Fig. 1 the relaxation of these integral parameters to a final configuration. The initial configuration is assumed to be spherical, so violent meridional motions begin. Moreover, because the equation of state is different from that used by Imshennik \& Nadyozhin (1992), we must modify the initial model slightly; it is not in exact equilibrium. So the meridional energy grows quickly from $E^{\mathbf{k} 0}=0$ at $t=0$ and reaches its maximum value (corresponding to $E^{\mathrm{k} 0} /\left|E^{\mathrm{gr}}\right| \simeq 0.08$ ) at $t \simeq 0.3 \mathrm{~ms}$. Later, the energy of these motions is quickly dissipated in shock waves. This dissipation leads to the growth of the thermal energy and to the decrease of $E^{\mathrm{k} 0}$ by more than a factor of ten in a time $\sim 6$ ms. Due to the overall expansion and conservation of angular momentum, the parameter $\eta=E^{\mathrm{rot}} /\left|E^{\mathrm{gr}}\right|$ also decreases from the initial value $\eta=0.42$ and stabilizes at the level $\eta=0.23$.

Fig. 2 demonstrates the shape of the final configuration. The stability of the rotating body thus obtained can be studied either in a linear analysis for this value of $\eta$ or it can be used as a starting point for 3-dimensional calculations of the fission.

Let us now briefly discuss the outstanding challenges for further research in the project. The problem of fission involves not only the hydrodynamical computations - it is necessary also to analyze the modes of the redistribution of angular momentum and the role of the magnetic fields in this process. The next point is the problem of the mass exchange under the influence of gravitational radiation. Of course, this secular process cannot be studied by a dynamic code, so special techniques and approximations must be developed. Finally, the problem of the explosion of a neutron star below the minimum stable mass deserves special attention. Recently, Colpi, Shapiro \& Teukolsky (1993) have found that the energy release by $\beta$-decays leads to an explosion with $E^{\mathrm{k}} \sim 0.5 \times 10^{50} \mathrm{ergs}$. This is an order of magnitude below than the value found by Blinnikov et al. (1990) in the more idealised approximation of the fully catalyzed matter. The numerical approach of Colpi et al. (1993) is more accurate than ours; still, improvements in their scheme are still possible. There also remain some interesting questions regarding physical processes: the evolution of a hot newborn neutron star may follow another path than that of an old 


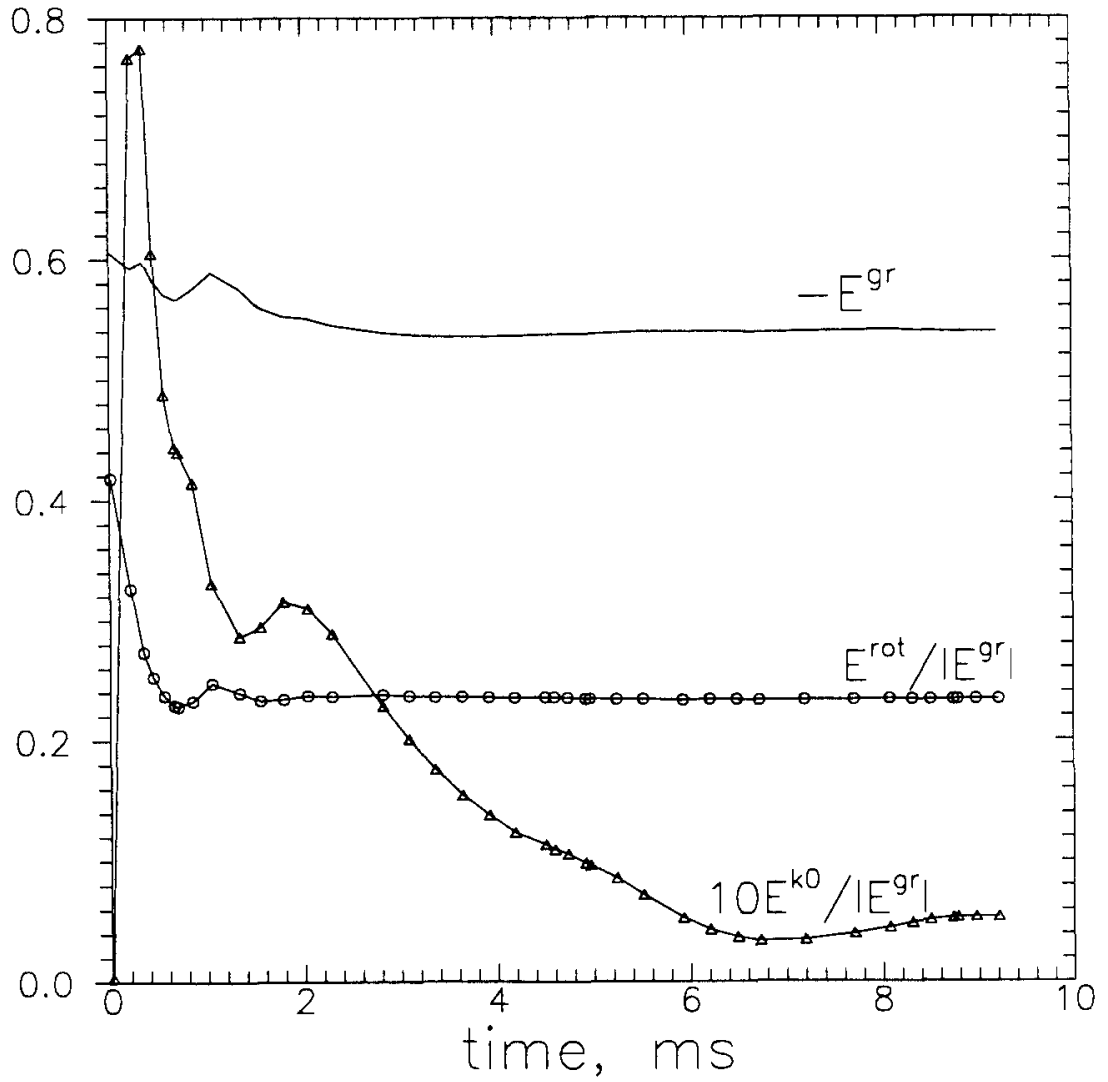

Figure 1. Evolution in time of $E^{\mathrm{gr}}$, in units $2 \times 10^{53} \mathrm{erg}$, and of the fractions $\eta=E^{\mathrm{rot}} /\left|E^{\mathrm{gr}}\right|$, $10 E^{\mathrm{k} 0} /\left|E^{\mathrm{gr}}\right|$.

cold one, as investigated in the cited papers; the descripton of neutrino transfer may be important, etc. If this "standard bomb" appears to be too weak to account for a normal SN II event, then one can consider a natural combination of this mechanism with the nuclear explosion in the layers overlying the core (Bodenheimer \& Woosley 1983). The thermonuclear explosion would be triggered then by the exploding neutron star.

\section{Theoretical light curves}

The second part of our contribution is devoted to another project which is being developed at ITEP and at Sternberg Astronomical Institute (in close collaboration with MPA, Garching and UC, Santa Cruz). This project involves the modelling of Type II SN light curves. We briefly describe some results of our computations of SN light curves accounting for multi-frequency-group time-dependent radiation transfer. The results are virtually independent of the details of the explosion mechanism, but they are sensitive to the explosion energy, to the structure of the outer layers of the progenitor, and to the amount of the radioactive ${ }^{56} \mathrm{Ni}$ produced. In our computations we use both simplified progenitor models and detailed evolutionary models constructed by Weaver \& Woosley (1993) and Woosley, Langer \& Weaver (1993). We use our code STELLA which incorporates implicit hydrodynamics coupled to a time-dependent multi-group non-equilibrium 


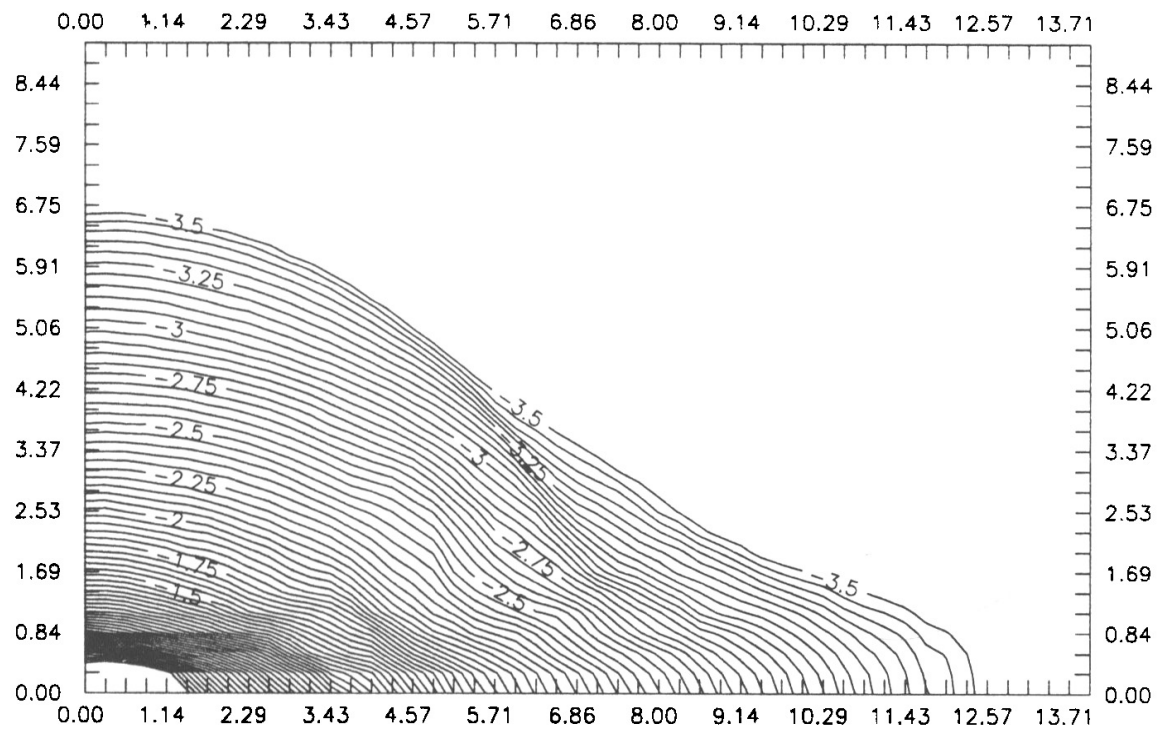

Figure 2. The isodensity contours for the final configuration obtained by Aksenov \& Imshennik (1994). The lines correspond to the averaged density $\lg \left(t_{\mathrm{fin}}^{-1} \int_{0}^{t_{\mathrm{fin}}} \rho(t) d t\right)$. The density is in units $10^{14} \mathrm{~g} / \mathrm{cm}^{3}$, and $r, z$ in $10^{6} \mathrm{~cm}$.

radiative transfer. For the present runs we use 200 Lagrangean mesh zones and 16 energy groups, spaced geometrically from $\lambda=10 \AA$ to $\lambda=5 \times 10^{4} \AA$.

The input physics in our code is the same as described in Blinnikov \& Bartunov (1993), but for low mass models or for the "tail" phase it is essential to take into account the gamma-ray transfer, which we ignored in our previous work. We have tried various prescriptions for the gamma-ray energy deposition and have chosen the "exact" numerical solution of the time-independent spherically symmetric gamma-ray transport in the one-group approximation, replacing the scattering of gamma-rays by an effective pure absorption with the opacity $\kappa_{\gamma}=0.03 \mathrm{~cm}^{2} / \mathrm{g}$ (Ambwani \& Sutherland 1988). We assume that positrons always deposit their energy locally. We take all constants for ${ }^{56} \mathrm{Ni}$ and ${ }^{56}$ Co decays from the compilation by Nadyozhin (1993).

We present in Fig. 3 examples of computed and observed $B$-light curves for linear type II SNe. Fig. 4 displays our results for a typical type II- P supernova. The models have different values of progenitor mass, $M_{0}$, radius, $R_{0}$ and hydrogen mass, $M_{\mathrm{H}}$, but they have the same assumed values for explosion energy, $E_{0}=2 \times 10^{51} \mathrm{erg}$, and ${ }^{56} \mathrm{Ni}$ mass, $M_{\mathrm{Ni}}=0.078 M_{\odot}$, approximately the standard parameters for SN 1987A.

Our set of hydrodynamical models shows that the observations of "normal" SNe II-P and II-L are well reproduced by the explosion of a supergiant progenitor with a radius of several hundreds $R_{\odot}$; but for SNe II-L one must assume a small hydrogen envelope mass $1-2 M_{\odot}$. The unusual brightness of some events, such as SN $1979 \mathrm{C}$, is naturally explained by re-radiation of ultraviolet photons created at shock breakout in the superwind shell surrounding the exploding star (Blinnikov \& Bartunov 1993). Both faint and very bright SN II-P and SN II-L events are explained without requiring large masses of radioactive material. We conclude that all type II supernovae (linear, plateau and 1987A subtypes) are probably produced by an explosion with one and the same energy and that all varieties 


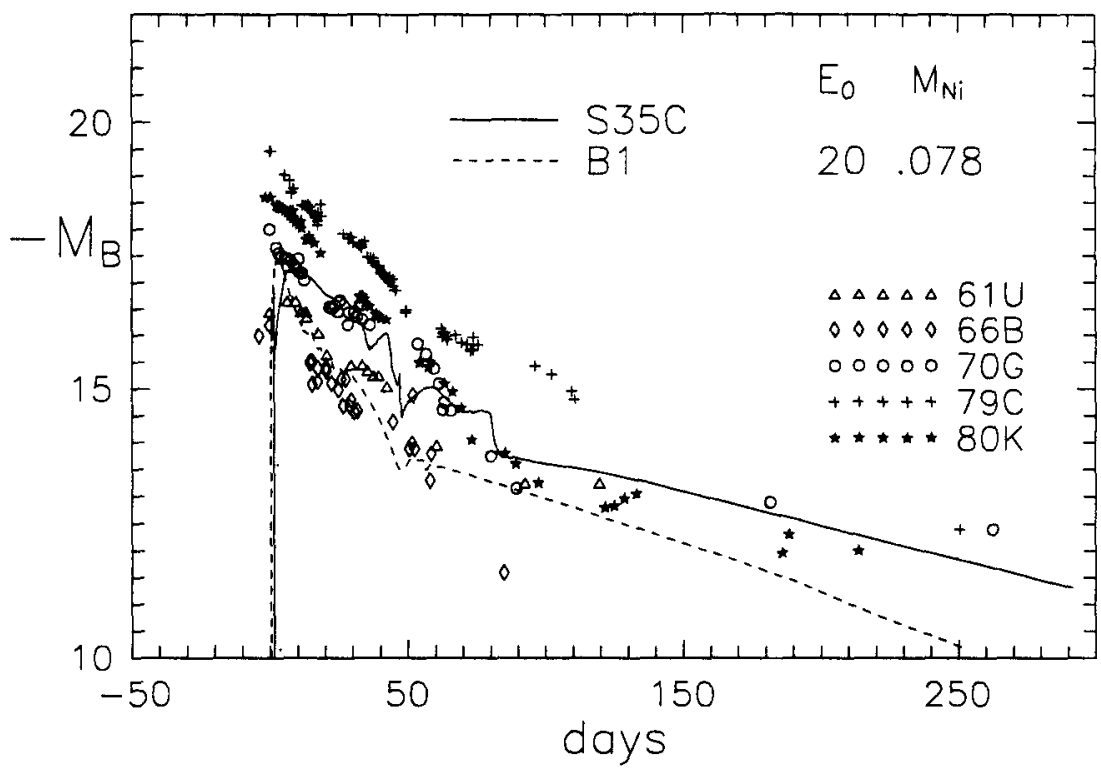

FIgURE 3. Theoretical light curves for SNe II-L. The solid line is for the pre-SN model s35c of Woosley et al. (1993) $M_{\mathrm{pSN}}=15.2 M_{\odot}, R_{0}=970 R_{\odot}, M_{\mathrm{H}}=1.44 M_{\odot}$. The dashed line is the model B1 of Blinnikov \& Bartunov (1993) $M_{\mathrm{pSN}}=7.4 M_{\odot}, R_{0}=600 R_{\odot}, M_{\mathrm{H}}=1.84 M_{\odot}$. The observational data for SNe II-L are compiled and reduced to one system by Bartunov et al. (1992).

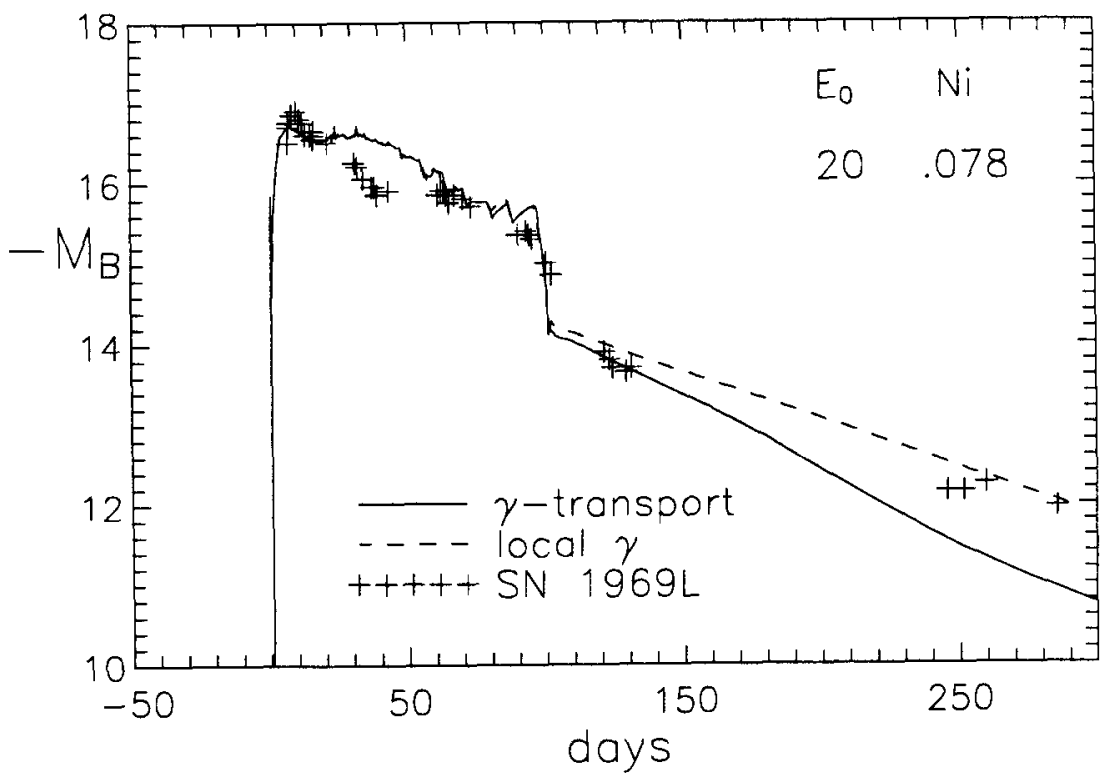

Figure 4. The $B$-light curve for SN 1969L of type II-P. The solid line is our prediction for the pre-SN model s15s7a of Weaver \& Woosley $(1993) M_{\mathrm{pSN}}=15.1 M_{\odot}, R_{0}=500 R_{\odot}, M_{\mathrm{H}}=6.9 M_{\odot}$. 
of light curves can be explained by the progenitor radius, by the density of the progenitor wind and by the amount of hydrogen left in the envelope.

It seems that the same is true also for the recent peculiar supernova SN 1993J discovered in NGC 3031 (Garcia 1993). As noted by Woosley (1993b), Nomoto et al. (1993) and Shigeyama et al. (1993), its light curve is similar to a "Type II-b" (Woosley et al. 1987; Woosley 1991). The evolution of the spectrum supports this classification (Filippenko \& Matheson 1993).

The type II-b progenitor is a normal type II progenitor which has lost almost all of its hydrogen envelope (Woosley 1991), so it is similar to type II-L (Swartz et al. 1991, Blinnikov \& Bartunov 1993). After comparison with other models from our set, we assume $R_{0}=200 R_{\odot}$ as initial condition (Bartunov et al. 1994). We chose $M=1.41 M_{\odot}$ for the mass cut of the core. The burst was produced by the release of the thermal energy $E_{0}$ in the mass range $1.41-1.44 M_{\odot}$ in ten seconds.

Here we present results for the same burst energy, $E_{0}=2 \times 10^{51} \mathrm{ergs}$, as in other models but with the lower ejecta mass, $M_{0}=3 M_{\odot}$, corresponding to progenitor mass $4.41 M_{\odot}$. The asymptotic kinetic energy is $1.9 \times 10^{51} \mathrm{erg}$ in our model, which of course depends on the position of the mass cut. The mass of hydrogen in our model is $M_{\mathrm{H}}=0.92 M_{\odot}$ and the chemical composition is assumed as in model B1 (Fig.2 in Blinnikov \& Bartunov 1993). The parameters of our model are similar to those in independent models by Shigeyama et al.(1993), Woosley \& Eastman (1993), Ray et al.(1993) and Utrobin (1993), although we assume higher $M_{\mathrm{H}}$ (especially than the first three papers).

Fig. 5 displays our computed light curve in the $B$ band for first 60 days and the observations of SN 1993J (compilation by Kato 1993). We assume the distance modulus 27.6 for M81 (Freedman 1990). In this plot it is assumed that the Supernova exploded on 1993, March, 26.5. Our results show that the explosion occurred a few hours before the first pre-discovery observation (Neely 1993) recorded on March 28.3, most probably, in the interval March 28.0-28.2. (See also the Fig. 6: the shape of the early light curve is more clear there.)

The discovery of X-ray radiation by ROSAT (Zimmermann et al. 1993) and ASCA (Tanaka 1993) as well as the detection of radio emission (Weiler et al. 1993, Weiler 1993) clearly demonstrate the presence of an extended circumstellar envelope, produced by a progenitor superwind. Panagia et al. (1993) estimate the pre-supernova mass loss rate as $\dot{M} \sim 2 \times 10^{-6} M_{\odot} / \mathrm{yr}$. We have studied the influence of a similar circumstellar envelope on the visible light in the same way as Blinnikov \& Bartunov (1993) who followed Grasberg $\&$ Nadyozhin (1987). We have assumed the value of $\dot{M}$ a factor of 4 higher than suggested by Panagia et al. (1993) and surrounded the model with $R_{0}=100 R_{\odot}$ by a superwind envelope with the density distribution $\rho \propto r^{-2}$. The outer radius of the envelope is fixed at $3 \times 10^{3} R_{\odot}$. For these parameters the photosphere is at $R_{\mathrm{ph}} \sim 200$. We compare the results for $M_{V}(t)$ for the first 3 days of our model $R_{0}=200 R_{\odot}$ without wind with that having the wind in Fig. 6. It is clear that the wind produces more sharp rise and some additional transient features in the light curve.

One of the most interesting conclusions of our modelling is that SNe II of quite different appearance (SN 1969L, SN 1987A, SNe II- L and now SN 1993J) can produced by explosions with the same energy within a factor of 2 . In most cases the amount of ${ }^{56} \mathrm{Ni}$ expelled is about the same. This result favors the existence of a universal explosion mechanism for SNe II.

We have no space to discuss here very interesting problems involved in modelling $\mathrm{SNe} \mathrm{Ib}$ wich are related to SNe II. We just note that we agree with the conclusion of Baron, Young \& Branch (1993) that it is difficult to have a very long radioactive "tail" 


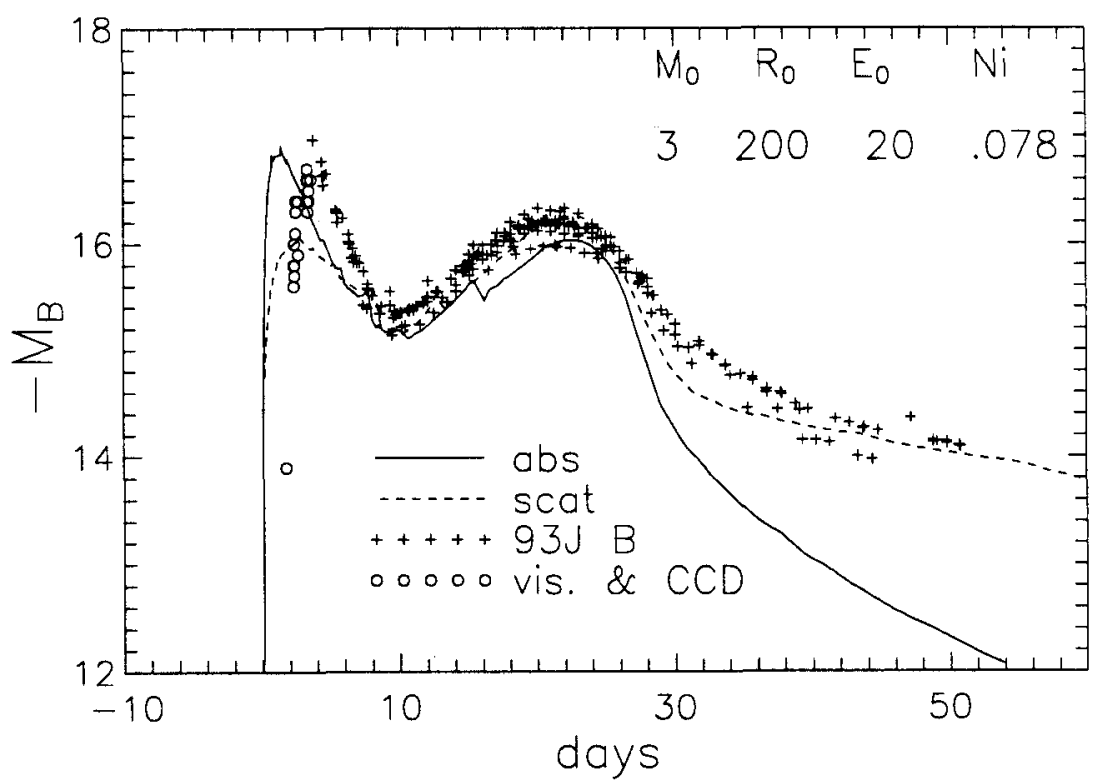

Figure 5. The absolute blue magnitude $M_{B}$. Pluses show the observed $M_{B}$ magnitudes of SN 1993J for the distance modulus 27.6 and circles correspond to visual and CCD estimates. The explosion is assumed to occur on March 26.5. The solid line is the theoretical light curve when the absorption is put equal to the total extinction and the dashed line is for the correct treatment of Thomson scattering.

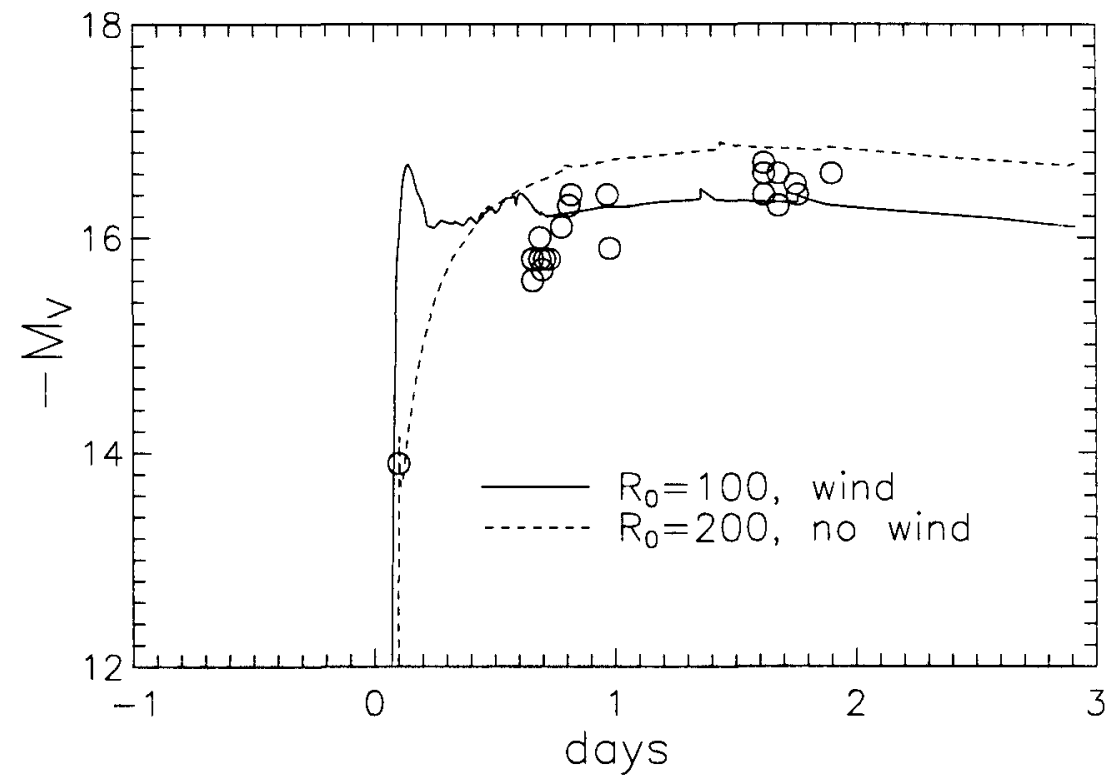

Figure 6. Comparison of $V$-light curves for the first 3 days for a model with the wind (solid), $R_{0}=100 R_{\odot}$, and the standard model $R_{0}=200 R_{\odot}$. Circles show the visual and CCD estimates. Now the explosion is assumed to occur on March 28.2. 
together with a narrow "dome" near maximum. We suggest that the discrepancy might be resolved by a shock wave, forming the "tail".

\section{Acknowledgements}

The work is partly supported by the Russian Foundation for Fundamental Research grants No 93-02-3637, 93-02-17114. Our visit to the Colloquium No 145 is supported by IAU and ISF. We are especially grateful to R.McCray for his help.

\section{REFERENCES}

Aksenov, A. G. 1993, About the Poisson equation solver. Preprint ITEP-45

Aksenov A. G. \& Imshennik, V. S. 1994, Pisma Astr. Zh. 20, 32.

Ambwani, K. \& Sutherland, P. G. 1988, ApJ, 325, 820

Baron, E., Young, T. R. \& Branch, D. 1993, ApJ, 409, 417

Bartunov, O. S., Makarova, I. N. \& Tsvetkov, D. Yu. 1992, private communication

Bartunov, O. S., Blinnikov, S. I., Pavlyuk., N. N. \& Tsvetkov, D. Yu. 1994, A Model for Supernova 1993J. A\&A, 281, L53

Bethe, H. \& Wilson, J. 1985, ApJ, 295, 14

Bisnovatyi-Kogan, G. S. 1970, Soviet Ast., 14, 625

Blinnikov, S. I. \& Bartunov, O. S. 1993, A\&A, 273, 106

Blinnikov, S. I., Novikov, I. D., Perevodchikova, T. V. \& Polnarev, A. G. 1984, Soviet Ast. Lett., 10,177

Blinnikov, S. I., Imshennik, V. S., Nadyozhin, D. K., Novikov, I. D., Perevodchikova, T. V. \& Polnarev, A. G. 1990, Soviet Ast. 34, 595

Bodenheimer, P. \& Woosley, S. 1983, ApJ, 269, 281.

Burrows, A. 1993, these proceedings.

Colella, P. \& Glas, H. M. 1985, J. Comp. Phys., 59, 264

Colella, P. \& Woodward, P. R. 1984, J. Comp. Phys., 54, 174

Colpi, M., Shapiro, S. L. \& Teukolsky, S. A. 1993, ApJ, 414, 717

Filippenko, A. V. \& Matheson, T. 1993, IAU Circ. No 5787

Freedman W. L. 1990, ApJ, 355, L35.

Friedmann, B. \& Pandharipande, V. R. 1981, Nucl. Phys. A, 361, 502

Garcia, F. 1993, IAU Circ. No. 5731.

Grasberg, E. K.,\& Nadyozhin, D. K. 1987, Soviet Ast., 31, 629

Hillebrandt, W., Müller, E. \& Mönchmeyer, R. 1990, in Nuclear Equation of State, pp 689-719, Plenum Press, New York.

Imshennik, V. S. 1992a, in Astrophysics on the Threshold of 21st Century, ed. N. S. Kardashev. Gordon \& Breach, Philadelphia.

Imshennik, V. S. 1992b, Soviet Ast., 18, 194

Imshennik, V. S. \& Nadyozhin, D. K. 1977, Soviet Ast., 3, 33

Imshennik, V. S. \& Nadyozhin, D. K. 1992, Soviet Ast. Lett. 18, 79

Janka, H.-Th. 1993, these proceedings.

Kato, T. 1993, SN 1993J photometry update. E-mail from Kyoto University, May 18th.

Meszaros, P. \& Rees, M. J. 1992, ApJ, 397, 570

Nadyozhin, D. K. 1977, Ap. \& Space Sci., 49, 399

Nadyozhin D. K. (1993). Preprint No. 61, Astronomical Institute University of Basel 
Narayan, R., Paczyński, B. \& Piran, T. 1992, ApJ, 395, L83

Neely, A. (1993). IAU Circ. No 5740.

Nomoto, K., Suzuki, T., Shigeyama, T., Kumagai, S., Yamaoka, H. \& Saio, H. 1993, Nature, 364,507

Panagia, N., Van Dyk, S. D., \& Weiler, K. W. 1993, IAU Circ. No 5762

Ray, A., Singh, K. P. \& Sutaria, F. K. 1993, J. Ap. \& Astr., 14, 53

Shigeyama, T., Suzuki, T., Kumagai, S., Nomoto, K., Saio, H. \& Yamaoka, H. 1994, ApJ, 420, 321

Shklovsky, I. S. 1970, Ap. Lett., 8, 101

Swartz, D. A., Wheeler, J. C. \& Harkness, R. P. 1991, ApJ, 374, 266.

Tanaka, Y. 1993, IAU Circ. No 5753.

Tassoul, J.-L. 1979, Theory of Rotating Stars, Princeton Univ. Press

von Weizsäcker, C. F. 1947, Zeitschrift für Astrophys., 24, 181

Utrobin, V. 1993, A\&A, 270, 249

Weaver, T. A.,\& Woosley, S. E. 1993, Phys. Reports, 227, 65

Weiler, K. W. 1993, Data on radio observation of SN 1993J, communicated via E-mail.

Weiler, K. W., Sramek, R. A., Van Dyk, S. D. \& Panagia N. 1993, IAU Circ. No 5752.

Woosley S. E. 1991, in Supernovae. Tenth Santa Cruz Workshop in Astr.Astrophys., ed. S. E. Woosley (New York: Springer Verlag), p.202.

Woosley S. E. 1993a, ApJ, 405, 273

Woosley S. E. (1993b). Private communication via E-mail of May, 9th.

Woosley, S. E., Langer, N. \& Weaver, T. A. 1993, ApJ, 411, 823

Woosley, S. E., Pinto, P. A., Martin, P. G. \& Weaver, T. A. 1987, ApJ, 318, 664

Woosley, S. E., Weaver, T. A. \& Eastman, R. G. 1993, these proceedings.

Zimmermann, H. U., Lewin, W., Magnier, E., Predehl, P., Hasinger, G., Pietsch, W., Aschenbach, B., Trümper, J., Fabbiano, G., van Paradijs, J., Lubin, L. \& Petre, R. 1993, IAU Circ. No 5748 